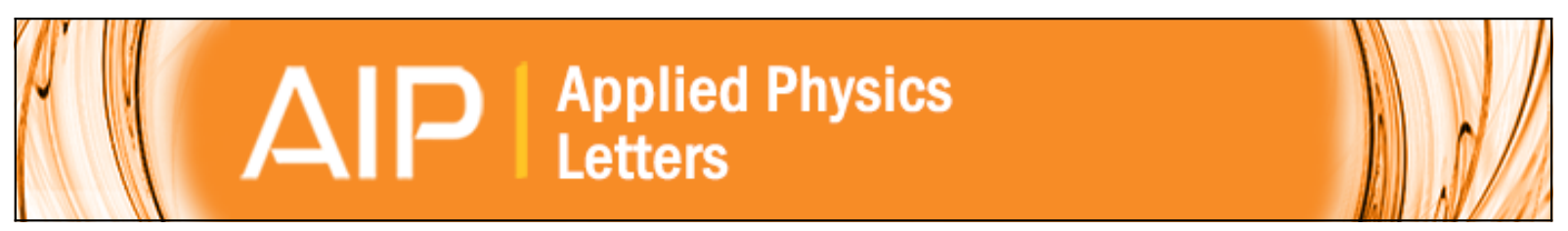

\title{
Design and characterization of ultra-stretchable monolithic silicon fabric
}

\author{
J. P. Rojas, A. Arevalo, I. G. Foulds, and M. M. Hussain
}

Citation: Applied Physics Letters 105, 154101 (2014); doi: 10.1063/1.4898128

View online: http://dx.doi.org/10.1063/1.4898128

View Table of Contents: http://scitation.aip.org/content/aip/journal/apl/105/15?ver=pdfcov

Published by the AIP Publishing

\section{Articles you may be interested in}

Materials and fabrication sequences for water soluble silicon integrated circuits at the $90 \mathrm{~nm}$ node

Appl. Phys. Lett. 106, 014105 (2015); 10.1063/1.4905321

Design and fabrication of a novel microgripper with four-point contact fingers

J. Vac. Sci. Technol. A 29, 011007 (2011); 10.1116/1.3520645

Four point bending setup for characterization of semiconductor piezoresistance

Rev. Sci. Instrum. 79, 044703 (2008); 10.1063/1.2908428

Curving monolithic silicon for nonplanar focal plane array applications

Appl. Phys. Lett. 92, 091114 (2008); 10.1063/1.2883873

Monolithically integrated low-loss silicon photonic wires and three-dimensional tapered couplers fabricated by self-profile transformation

Appl. Phys. Lett. 91, 191114 (2007); 10.1063/1.2809359

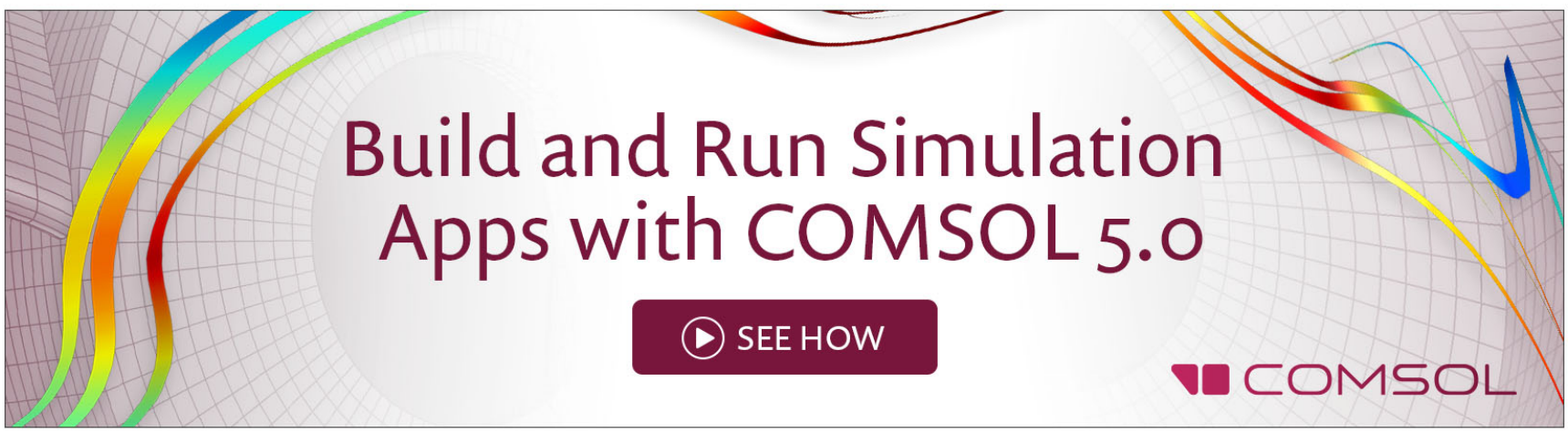




\title{
Design and characterization of ultra-stretchable monolithic silicon fabric
}

\author{
J. P. Rojas, ${ }^{1,2}$ A. Arevalo, ${ }^{2,3}$ I. G. Foulds, ${ }^{3,4}$ and M. M. Hussain ${ }^{1,2, a)}$ \\ ${ }^{1}$ Integrated Nanotechnology Lab, King Abdullah University of Science and Technology, 23955 Thuwal, \\ Saudi Arabia \\ ${ }^{2}$ Computer, Electrical and Mathematical Sciences \& Engineering Division, King Abdullah University of \\ Science and Technology, 23955 Thuwal, Saudi Arabia \\ ${ }^{3}$ Electromechanical Microsystems \& Polymer Integration Research Lab, King Abdullah University of Science \\ and Technology, 23955 Thuwal, Saudi Arabia \\ ${ }^{4}$ School of Engineering, The University of British Columbia, Okanagan Campus, Kelowna, VIV IV7 British \\ Columbia, Canada
}

(Received 15 August 2014; accepted 19 September 2014; published online 13 October 2014)

\begin{abstract}
Stretchable electronic systems can play instrumental role for reconfigurable macro-electronics such as distributed sensor networks for wearable and bio-integrated electronics. Typically, polymer composite based materials and its deterministic design as interconnects are used to achieve such systems. Nonetheless, non-polymeric inorganic silicon is the predominant material for $90 \%$ of electronics. Therefore, we report the design and fabrication of an all silicon based network of hexagonal islands connected through spiral springs to form an ultra-stretchable arrangement for complete compliance to highly asymmetric shapes. Several design parameters are considered and their validation is carried out through finite element analysis. The fabrication process is based on conventional microfabrication techniques and the measured stretchability is more than $1000 \%$ for single spirals and area expansions as high as 30 folds in arrays. The reported method can provide ultra-stretchable and adaptable electronic systems for distributed network of high-performance macro-electronics especially useful for wearable electronics and bio-integrated devices. (C) 2014 AIP Publishing LLC. [http://dx.doi.org/10.1063/1.4898128]
\end{abstract}

With the emergence of Internet of Things, flexible and stretchable electronics are gaining more and more scientific and commercial relevance due to their contribution to the expanding wearable industry, where compliance, weight, and coverage play a very important role. Novel ways to engineer materials are constantly being envisioned in order to fulfill the requirements of a light-weight, tunable-shape, and conformable electronic system. ${ }^{1,2}$ On the other hand, $90 \%$ of electronics in our daily life have silicon as the foundational block. Moreover, the big data paradigm demands ever-growing processing and storage capabilities, which are possible to reliably accomplish through state-of-the-art silicon technologies only. Starting on the basis that silicon industry has evolved to become the main paradigm that constantly and exponentially pushes technology towards new frontiers, the rising challenge is now to overcome its planar, rigid, and brittle nature. Looking forward, stretchable and easily deployable silicon based integrated circuits (ICs) will facilitate the development of many new essential technologies, such as bio-integrated systems for monitoring, diagnosis, and even treatment.

Researchers have developed organic/polymer-based electronics with the promise of excellent mechanical properties and cost advantages. Although great advancements have been made on display technologies and great flexibility and stretchability can be achieved, the electrical properties of organic/polymeric-based devices are still far from silicon standards, with several orders of magnitude lower mobilities (the highest reported being in the order of $10^{1}$ compared to $10^{3} \mathrm{~cm}^{2} / \mathrm{V} \mathrm{s}$ for silicon). ${ }^{3-7}$ Furthermore, temperature

\footnotetext{
${ }^{\text {a) }}$ Author to whom correspondence should be addressed. Electronic mail: muhammadmustafa.hussain@kaust.edu.sa.
}

stability highly restricts processing and applications. With similar mechanical properties, composites of carbon nanotubes (CNTs) or other nanostructured semiconducting materials (such as $\mathrm{ZnO}$ nanowires) can achieve very high expansion ratios in the order of $10 \%-50 \%$, but again, electrical performance needs considerable further improvement to achieve high volume manufacturing demand. ${ }^{8-10}$ On the other hand, 2-dimensional materials show promising electrical and mechanical properties. Graphene-based devices have been developed to maintain operation even at 5\% stretching with electron and hole mobility in the order of $10^{2}$ and $10^{3}$ $\mathrm{cm}^{2} / \mathrm{V}$ s, respectively. ${ }^{11}$ Similarly, higher strains have been achieved (20\%) with a mobility of near $40 \mathrm{~cm}^{2} / \mathrm{V} \mathrm{s.}{ }^{12}$ Nevertheless, graphene's inherent semi-metallic nature restricts the use of graphene to RF applications. Recently, transfer printing technology has arrived to help overcoming the mechanical restrictions of many inorganic substrates and the electrical limitations of polymers. Electronic devices can be fabricated on inorganic substrates to achieve high performance, then be released in the form of nano/micro membranes or ribbons and subsequently transferred onto elastomeric substrates, which serve as mechanical support to attain flexibility and stretchability. ${ }^{13-16}$ Some recent developments show innovative designs to achieve high stretchability with inorganic materials such as metals and semiconductors. Serpentine metallic or fractal structures were introduced to achieve remarkable stretchable capabilities up to $2300 \%$ for metal interconnects sandwiched between polymers and up to $100 \%$ for silicon nanomembranes bonded onto a pre-strained elastomeric substrate. ${ }^{17-19}$ The area of expansion for these technologies remains in attaining the level of ultra large scale integration 
(ULSI), necessary to deal with the highly demanding protocols in big data, cloud computation, and neuromorphic or brain-inspired computation, where light and mechanical flexible substrates will become a game changer in terms of area efficiency and multi-functional capabilities.

Back in 2007, Peumans et al. proposed an interesting design where monolithic silicon circular islands were circled and interconnected through spiral springs achieving outstanding stretchability with an area expansion up to 51 times the original area. ${ }^{20}$ The islands can be used to host electronic components and the spiral springs act as both mechanical and electrical connections between islands. Inspired by that initial work, we have expanded the concept to design and fabricate arrays of hexagonal silicon structures connected with double-arm spirals, thus forming stretchable arrangements for full compliance to highly irregular shapes. Our design differs from Peumans et al. on the incorporation of a honeycomb-based arrangement for improved area efficiency, featuring larger hexagons for ICs implementation and achieving stretchable capabilities through spiral connectors, which can also be used for electric connection between hexagons. One very important consideration during the design is that in order to maintain a maximum allowable strain of $1 \%$, the arms should be 50 times thinner than the radius of the inner circle. This can be easily determined from the nominal strain definition of a strained beam,

$$
\varepsilon_{M A X}=\frac{t}{2 R},
$$

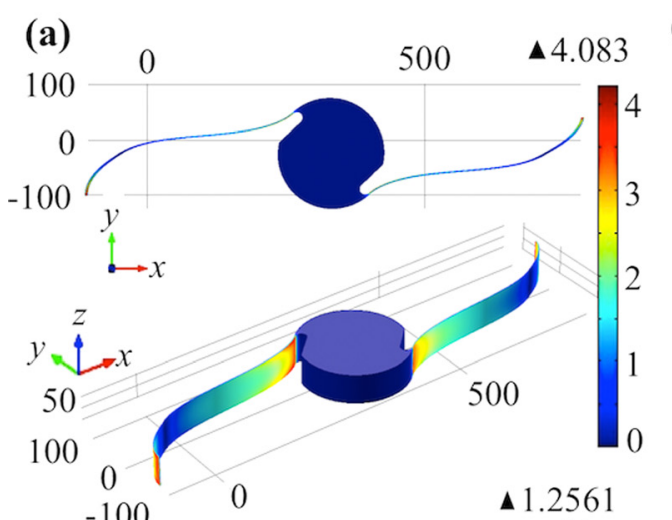

(b)
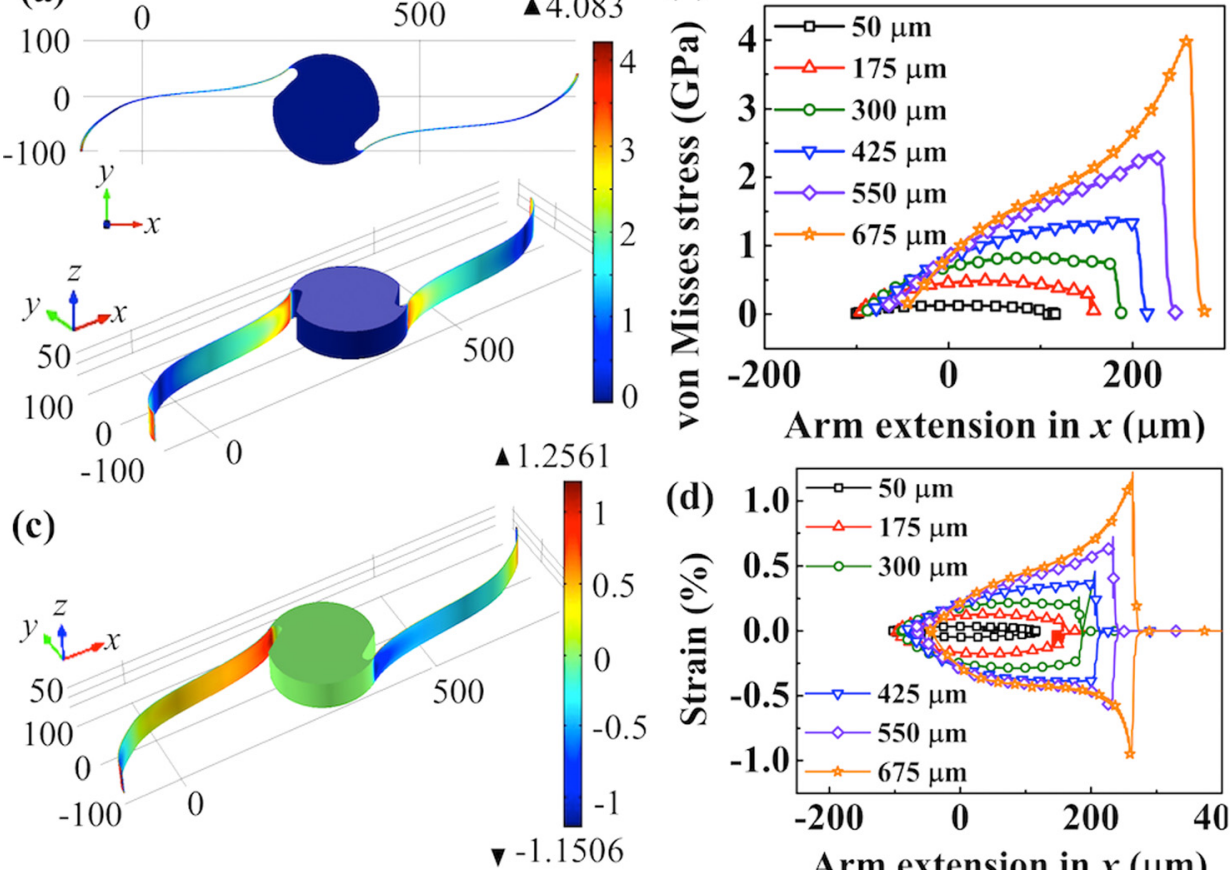

(e)

А 3.837

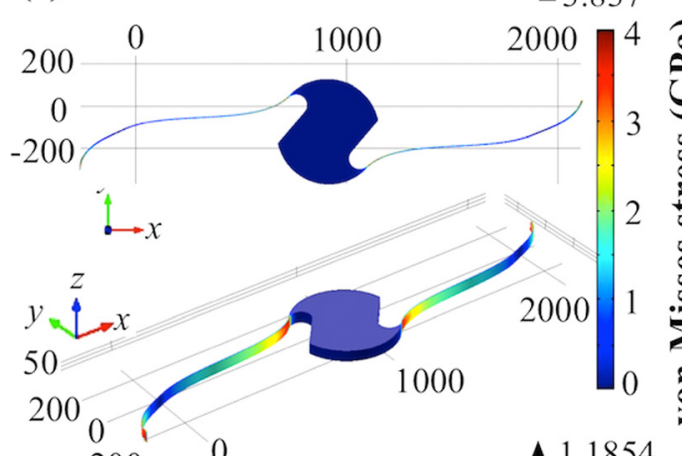

$\Delta 1.1854$

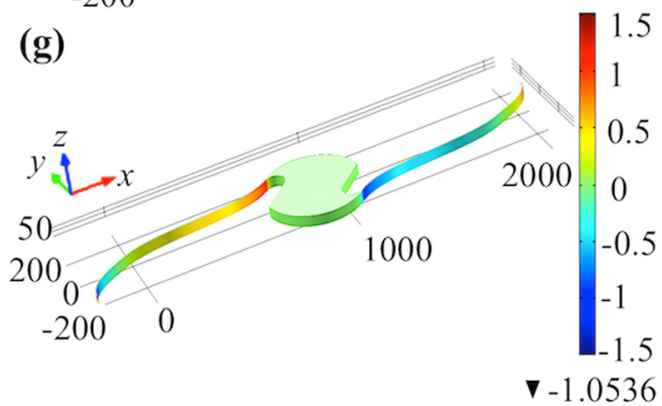

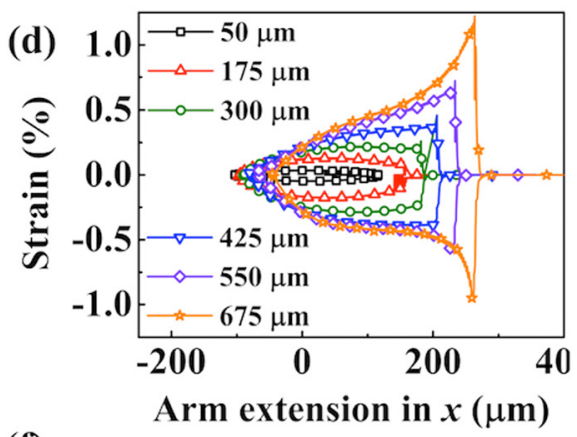

(f)

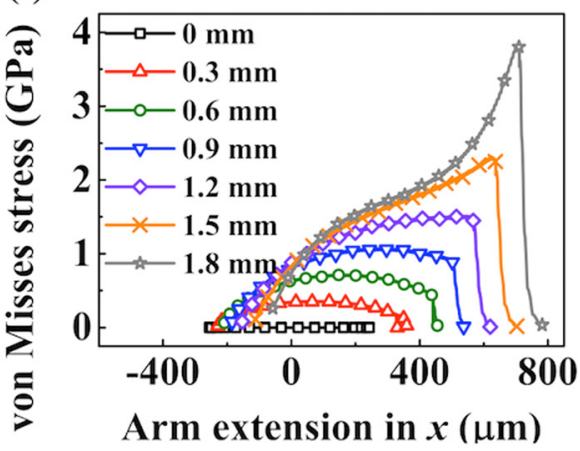

(h)

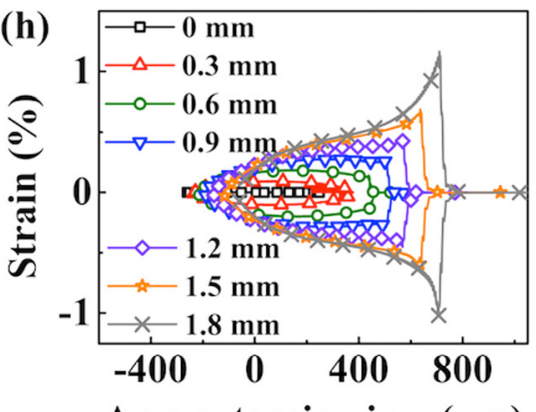

FIG. 1. Finite element analysis of ((a), (b), (e), (f)) von Mises stress and ((c), (d), (g), (h)) strain distributions along a single extended spiral's arm. (a)-(d) corresponds to a $2 \mu \mathrm{m}$-arm spiral extended to a horizontal displacement of $675 \mu \mathrm{m}$ and (e)-(h) to a $5 \mu \mathrm{m}$-arm spiral extended to $1.8 \mathrm{~mm}$. Axes units in (a), (c), (e), (g) are [ $\mu \mathrm{m}]$. 
where $t$ is the thickness of the spiral's arm and $R$ is the radius of the inner circle. Two arm-sizes were thus tested, $2 \mu \mathrm{m}$ and $5 \mu \mathrm{m}$ wide arms. Additionally, in an effort to increase robustness, serpentine shaped springs were added at the hexagonspiral connections to improve flexibility. Finite element simulations are used to validate the functionality of the spiral-based stretching scheme and serpentine connections. Stretchability of single spirals and arrays arrangements was evaluated showing exceptional results. Several designs were considered having in mind diverse dimensions and the use of single or double spirals per hexagon connection. The use of two spirals can be critical for back-up operation in case one of the springs may fail at any point.

In order to validate the chosen designs, we have performed finite element analysis to evaluate their strain distribution and identify possible weak points in the structures. Since we want to focus on the behavior of single spirals only, hexagon islands were omitted for simplicity. Fig. 1 shows the simulation results of fully extended single-turn spirals with the corresponding strain and stress distribution in the structures with very similar results between $2 \mu \mathrm{m}$ and $5 \mu \mathrm{m}$-arms spirals. A detailed description of the finite element simulation can be found in the supplementary material. ${ }^{21}$ The maximum strain and stress point are found at the beginning of the arms and in both cases is around 1.2\% (Figs. 1(a) and 1(e)). Von Mises stress is also shown with a maximum around $4000 \mathrm{MPa}$, much lower than the ultimate tensile strength of the silicon (Figs. 1(c) and 1(g)). Graphs in Figs. 1(b), 1(d), 1(f), and 1(h) illustrate the strain and stress distribution along the arms for different displacements of the whole structure, showing a faster increment towards high displacements as it would be expected. ${ }^{21}$

Furthermore, we noticed that especially high strains might appear at the base of the arms and therefore we decided to include serpentine-shaped springs to help mitigating this effect. Fig. 2 shows the comparison between arms with and without the serpentines at a fixed displacement of near $200 \mu \mathrm{m}$ in $\mathrm{X}$ direction. Thanks to the presence of the serpentine spring, the force needed to achieve such

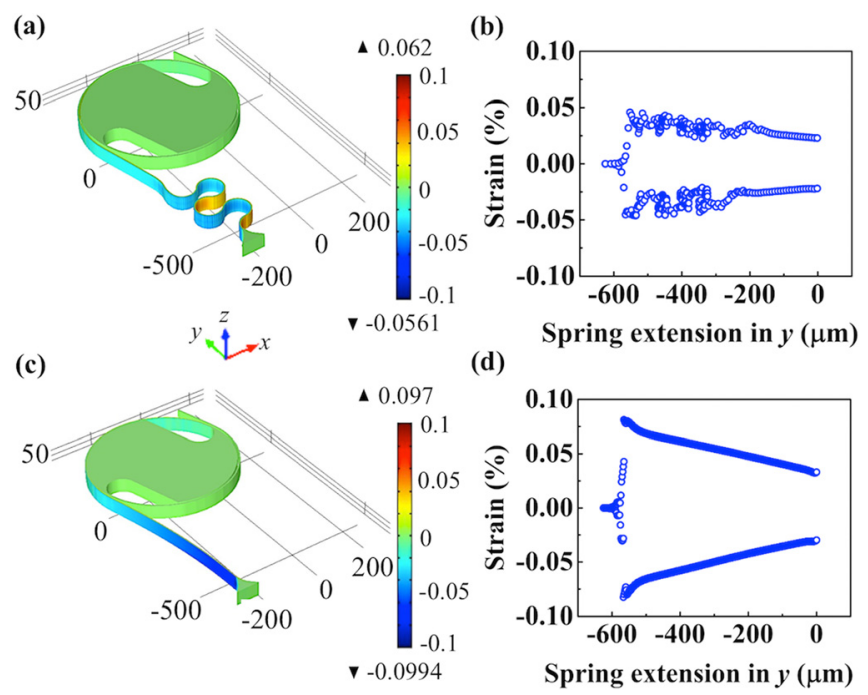

FIG. 2. Finite element analysis of strain distribution along spring structures ((a) and (b)) with and ((c) and (d)) without serpentine ending, for the same displacement of $200 \mu \mathrm{m}$ in X. Axes units in (a), (c) are $[\mu \mathrm{m}]$.

displacement was halved (from $60 \mu \mathrm{N}$ for a straight arm to $30 \mu \mathrm{N}$ for the one with the serpentine spring). Moreover, the strain developed was also halved and more evenly distributed along the spring, instead of concentrating the stress on a single point where it is more prompt to fail (Figs. 2(b) and 2(d)).

Once the designs were validated, we carried on with a simple 5-steps fabrication process flow, which is summarized in Fig. 3. We started with a comparatively expensive (than bulk monocrystalline silicon) silicon-on-insulator (SOI) substrate with $50 \mu \mathrm{m}$ silicon on top of $10 \mu \mathrm{m}$ silicon dioxide. First, we sputtered a thin film of Ti/Au $(20 \mathrm{~nm} / 300 \mathrm{~nm})$ (Fig. $3(\mathrm{a})$ ), which is then patterned through photolithography (Fig. $3(\mathrm{~b})$ ) and ion milling ( 4 cycles, $1.5 \mathrm{~min} / \mathrm{cycle}, 30 \mathrm{~s}$ breaks, 40 sccm Ar, 10 mTorr, $150 \mathrm{~W}_{\mathrm{RF}}, 300 \mathrm{~W}_{\mathrm{ICP}}$ ) to define the

(a) Deposit Ti/Au on SOI

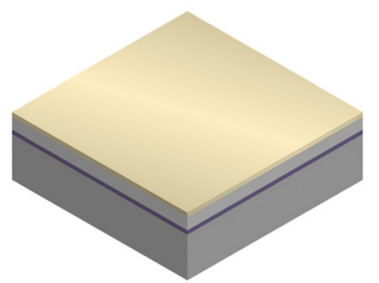

(c) Patterning of Ti/Au

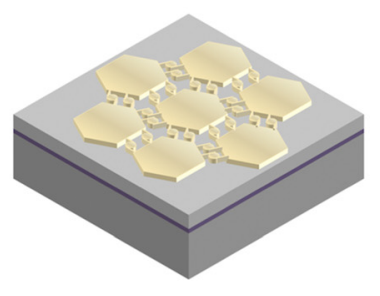

(d) DRIE of Si and Ti/Au removal

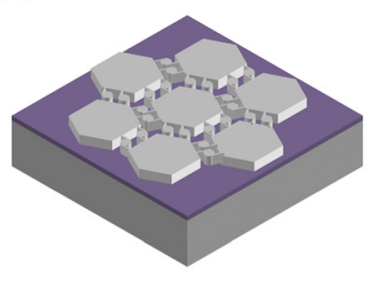

(e) Si structures release with VHF
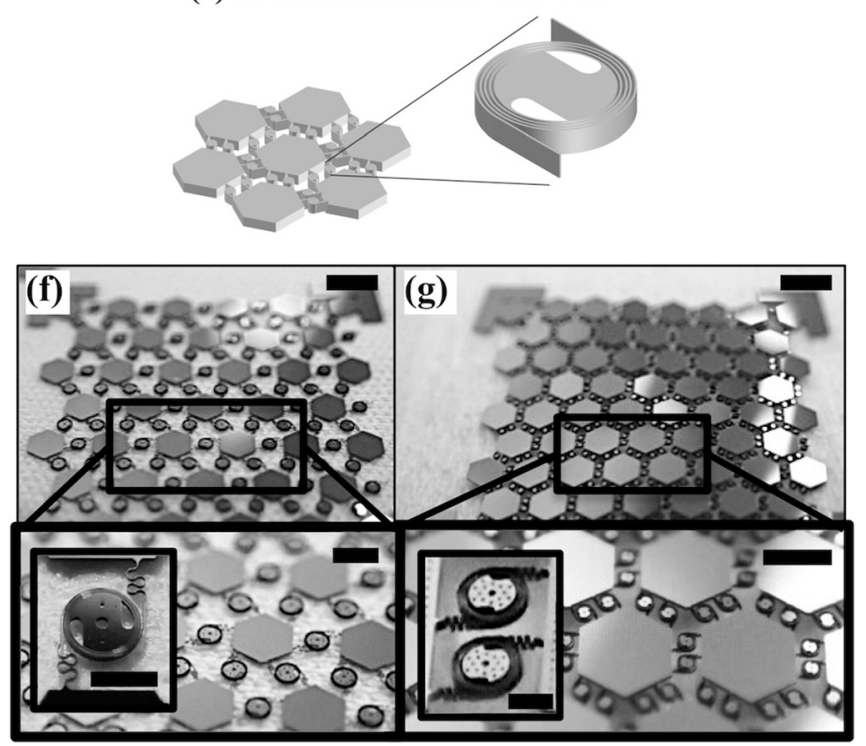

FIG. 3. Summarized fabrication process flow with digital photographs of final designs. (a) Hard mask deposition. (b) Photolithography step. (c) Hard mask's patterning. (d) Silicon DRIE and hard mask removal. (e) Release of silicon structures with VHF. (f) Digital photographs and zoom-ins of an array of $800 \mu \mathrm{m}$-side-hexagons interconnected by single $5 \mu \mathrm{m}$-arm spirals (scale bar is $2 \mathrm{~mm}$ long, $1 \mathrm{~mm}$ for the first zoom-in and $0.5 \mathrm{~mm}$ for the spiral zoom-in). (g) Digital photographs and zoom-ins of an array of $800 \mu \mathrm{m}$-sidehexagons interconnected by double $2 \mu \mathrm{m}$-arm spirals (scale bar is $2 \mathrm{~mm}$ long, $0.5 \mathrm{~mm}$ for the first zoom-in and $0.2 \mathrm{~mm}$ for the spiral zoom-in). 
hexagons and spirals (Fig. 3(c)). Etching holes were also formed to facilitate the final release step. Next, the $50 \mu \mathrm{m}$ silicon layer is etched down to the silicon dioxide level, using the metal as hard mask with a deep reactive ion etching (DRIE) tool (Fig. 3(d)). The metal hard mask is removed with wet gold and titanium etchant and finally the silicon structures are released in vapor hydrofluoric acid (VHF) at $40^{\circ} \mathrm{C}$ for around $60 \mathrm{~min}$ (Fig. 3(e)). Figs. 3(f) and 3(g) also illustrate digital photographs of arrays of single $5 \mu \mathrm{m}$-arms spiral connections and double $2 \mu \mathrm{m}$-arms spiral connections with zoom-ins. Devices could be accommodated in the large area hexagons (side length in hexagons is $800 \mu \mathrm{m}$ in Figs. 3(f) and 3(g)) as we have previously demonstrated, even in the presence of etching holes. $^{22-29}$ Additionally, the metal layer can be kept on the spiral structures to act as electric interconnects or to be the seed for a larger deposition of metal so as to reduce resistance.

Once released, we first characterized a single $5 \mu \mathrm{m}$-arm spiral (Fig. 4(a)) and double $2 \mu \mathrm{m}$-arm spirals (Fig. 4(c)). From the figures, we estimated outstanding stretchabilities of 945.5\% (hexagons with a single $5 \mu \mathrm{m}$-spiral, initial length: $1.1 \mathrm{~mm}$, final length: $\sim 11.5 \mathrm{~mm}$ ) and $1377.3 \%$ (squares with double $2 \mu \mathrm{m}$-spirals, initial length: $440 \mu \mathrm{m}$, final length: $\sim 6.5 \mathrm{~mm}$ ). The fact that the smaller spirals achieved a higher stretch ratio is actually due to the designed initial and final lengths, rather than mechanical differences in the structures. Thus, it is actually possible to produce structures with much higher stretch ratios or with specific ones, depending on application. Additionally, we present a supplementary movie showing the manual stretching of a single $5 \mu \mathrm{m}$-arm spiral for 8 cycles, which demonstrates mechanical robustness and reliability. ${ }^{21}$

With the aim of testing larger distributed network, we used a common inflatable balloon attached to a controllable nitrogen input. Handling remained the main challenge and although we fabricated large arrays as shown in Fig. 3, only smaller arrays were tested to facilitate attachment of the individual hexagons. Fig. 4(b) shows an array of 3 hexagons with $5 \mu \mathrm{m}$-arm spirals, each of which was expanded up to about $170 \%$ a total of 7.3 times the area. Fig. 4(d) shows a similar array but with double $2 \mu \mathrm{m}$-arm spirals, for a spiral expansion of $450 \%$, which is equivalent to an increment 30.25 times the area. Finally, a larger array with 7 hexagons and double $2 \mu \mathrm{m}$-arm spirals is depicted in Fig. 4(e). The
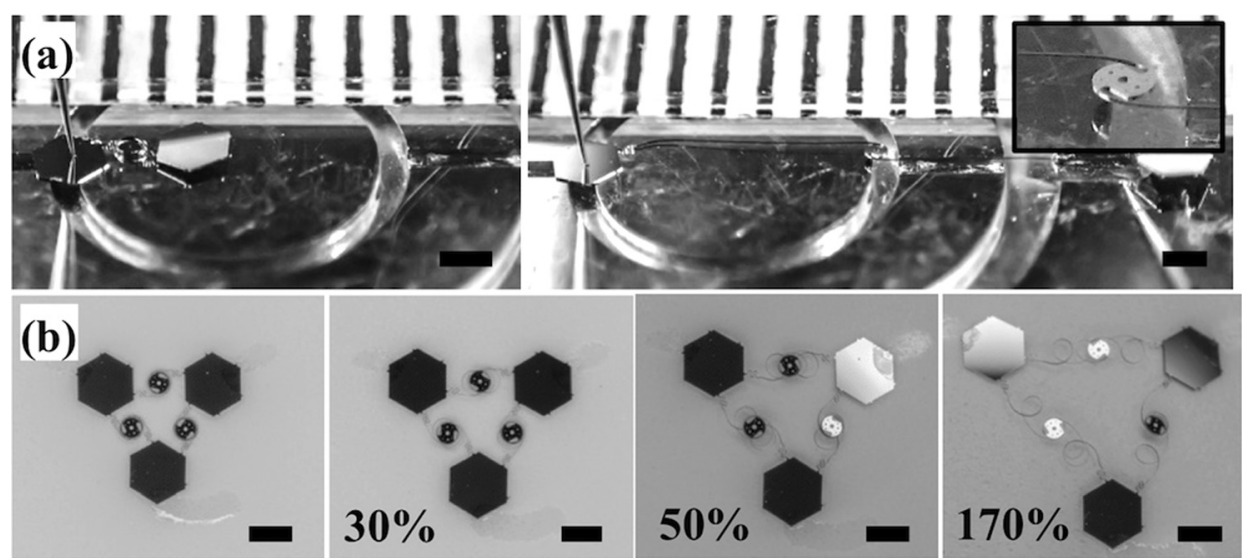
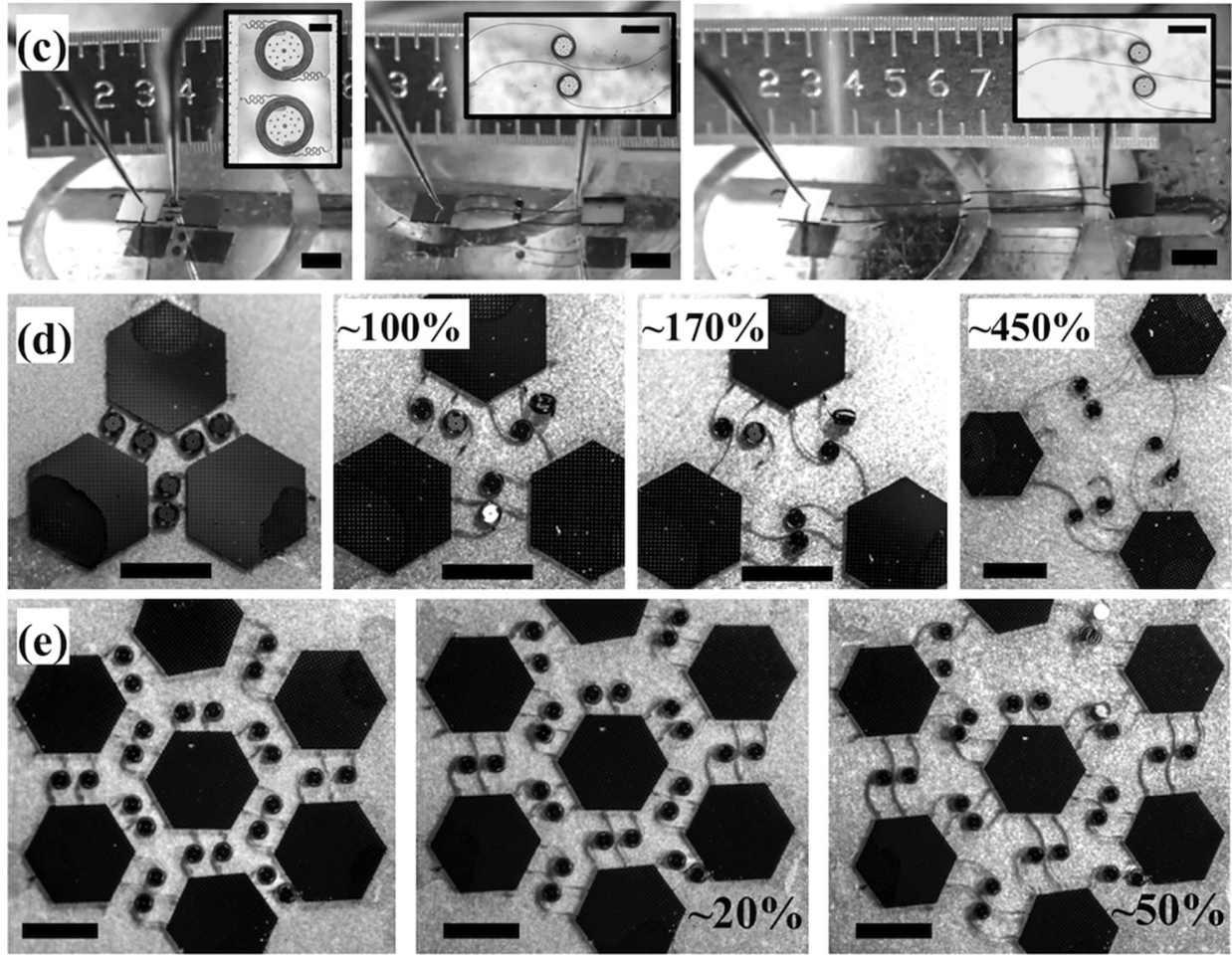

FIG. 4. (a) Full extension test of a $5 \mu \mathrm{m}$-arm spiral. (b) Area expansion test with an array of 3 hexagons connected with $5 \mu \mathrm{m}$-arm spirals. (c) Full extension test of double $2 \mu \mathrm{m}$-arm spirals (scale bars are $100 \mu \mathrm{m}$ for first inset and $500 \mu \mathrm{m}$ for second and third insets). (d) Area expansion test with an array of 3 hexagons connected with double $2 \mu \mathrm{m}$-arm spirals. (d) Area expansion test with an array of 7 hexagons connected with double $2 \mu \mathrm{m}$-arm spirals. Scale bars are $1 \mathrm{~mm}$ long unless otherwise stated. 
spirals were expanded up to $50 \%$, which equates to an area 2.25 times larger. It is important to remark the use of double spirals since they may fail, as observed in the figures, and backing them up with additional spirals is a key feature. Failure point was mostly presented at the end or beginning of the spirals as expected from the simulations. Nevertheless, we observed that in some cases the failure occurs along the arms, which can be explained given the presence of defects during the photolithography step. Such defect can then propagate vertically down the arm during the DRIE process and thus can cause irregular, weaker points along the spiral's length. Peumans et al. arrived to the same conclusion that smooth and defect-free arms work better and can tolerate even higher strain conditions. ${ }^{20}$ Additionally, we also observed un-wrapping of the spirals out of the XY plane, in which case the central disk tends to rotate out of plane and eventually it can lead to sooner failure along the arms. Consequently, finding a way to restrict the central disks' movement could help improving the mechanical reliability of the arrays.

In summary, we have presented an area efficient design to achieve ultra-stretchability in monolithic single-crystal silicon, a material with extraordinary mechanical and electrical properties that has leveraged today's technological era. The design was first studied through finite element analysis validating its mechanical robustness. The fabrication process is simple to follow and the measured stretchability was as elevated as $\sim 1000 \%$ for single spirals and area expansions as high as 30 folds in arrays. Handling remains a challenge but the outstanding results show a very promising opportunity to develop ultra-stretchable and adaptable systems for distributed network of macro-electronics focusing on wearable electronics and bio-integrated devices.

Research reported in this publication was supported by the KAUST Office of Competitive Research Grant 1: CRG1-2012-HUS-008.

${ }^{1}$ J. A. Rogers, T. Someya, and Y. Huang, Science 327, 1603 (2010).

${ }^{2}$ S. J. Benight, C. Wang, J. B. H. Tok, and Z. Bao, Prog. Polym. Sci. 38, 1961 (2013).

${ }^{3}$ B. Crone, A. Dodabalapur, Y. Y. Lin, R. W. Filas, Z. Bao, A. LaDuca, R. Sarpeshkar, H. E. Katz, and W. Li, Nature 403, 521 (2000).

${ }^{4}$ M. Kaltenbrunner, M. S. White, E. D. Głowacki, T. Sekitani, T. Someya, N. S. Sariciftci, and S. Bauer, Nat. Commun. 3, 770 (2012).

${ }^{5}$ T. Someya, Y. Kato, T. Sekitani, S. Iba, Y. Noguchi, Y. Murase, H. Kawaguchi, and T. Sakurai, Proc. Natl. Acad. Sci. U.S.A. 102, 12321 (2005).
${ }^{6}$ T. Sekitani and T. Someya, Adv. Mater. 22, 2228 (2010).

${ }^{7}$ Y. Yuan, G. Giri, A. L. Ayzner, A. P. Zoombelt, S. C. B. Mannsfeld, J. Chen, D. Nordlund, M. F. Toney, J. Huang, and Z. Bao, Nat. Commun. 5, 3005 (2013).

${ }^{8}$ T. Takahashi, K. Takei, A. G. Gillies, R. S. Fearing, and A. Javey, Nano Lett. 11, 5408 (2011).

${ }^{9}$ J. S. Bendall, I. Graz, and S. P. Lacour, ACS Appl. Mater. Interfaces 3, 3162 (2011)

${ }^{10}$ F. Xu, M.-Y. Wu, N. S. Safron, S. S. Roy, R. M. Jacobberger, D. J. Bindl, J.-H. Seo, T.-H. Chang, Z. Ma, and M. S. Arnold, Nano Lett. 14, 682 (2014).

${ }^{11}$ S.-K. Lee, B. J. Kim, H. Jang, S. C. Yoon, C. Lee, B. Hee Hong, J. A. Rogers, J. H. Cho, and J.-H. Ahn, Nano Lett. 11, 4642 (2011).

${ }^{12}$ S. H. Chae, W. J. Yu, J. J. Bae, D. L. Duong, D. Perello, H. Y. Jeong, Q. H. Ta, T. H. Ly, Q. A. Vu, M. Yun, X. Duan, and Y. H. Lee, Nature Mater. 12, 403 (2013).

${ }^{13}$ Y. Sun, W. M. Choi, H. Jiang, Y. Y. Huang, and J. A. Rogers, Nat. Nanotechnol. 1, 201 (2006).

${ }^{14}$ D.-Y. Khang, H. Jiang, Y. Huang, and J. A. Rogers, Science 311, 208 (2006).

${ }^{15}$ D.-H. Kim, J. Xiao, J. Song, Y. Huang, and J. A. Rogers, Adv. Mater. 22, 2108 (2010).

${ }^{16}$ X. Hu, P. Krull, B. d. Graff, K. Dowling, J. A. Rogers, and W. J. Arora, Adv. Mater. 23, 2933 (2011).

${ }^{17}$ Y. Zhang, H. Fu, S. Xu, J. A. Fan, K.-C. Hwang, J. Jiang, J. A. Rogers, and Y. Huang, J. Mech. Phys. Solids 72, 115 (2014).

${ }^{18}$ S. Xu, Y. Zhang, J. Cho, J. Lee, X. Huang, L. Jia, J. A. Fan, Y. Su, J. Su, H. Zhang, H. Cheng, B. Lu, C. Yu, C. Chuang, T.-i. Kim, T. Song, K. Shigeta, S. Kang, C. Dagdeviren, I. Petrov, P. V. Braun, Y. Huang, U. Paik, and J. A. Rogers, Nat. Commun. 4, 1543 (2013).

${ }^{19}$ J. A. Fan, W.-H. Yeo, Y. Su, Y. Hattori, W. Lee, S.-Y. Jung, Y. Zhang, Z. Liu, H. Cheng, L. Falgout, M. Bajema, T. Coleman, D. Gregoire, R. J. Larsen, Y. Huang, and J. A. Rogers, Nat. Commun. 5, 3266 (2014).

${ }^{20}$ K. Huang, R. Dinyari, G. Lanzara, J. Y. Kim, J. Feng, C. Vancura, F.-K. Chang, and P. Peumans, in IEEE International Electron Devices Meeting, 2007, 10-12 December 2007, pp. 217-220.

${ }^{21}$ See supplemental material at http://dx.doi.org/10.1063/1.4898128 for a detailed description of the finite element simulation and a movie of manual stretching of $5 \mu \mathrm{m}$-arm spiral for 8 cycles.

${ }^{22}$ J. P. Rojas, M. T. Ghoneim, C. D. Young, and M. M. Hussain, IEEE Trans. Electron. Devices 60, 3305 (2013).

${ }^{23}$ J. P. Rojas and M. M. Hussain, Phys. Status Solidi RRL 7, 187 (2013).

${ }^{24}$ J. P. Rojas, G. Torres Sevilla, and M. M. Hussain, Appl. Phys. Lett. 102, 064102 (2013).

${ }^{25}$ J. P. Rojas, A. Syed, and M. M. Hussain, in 2012 IEEE 25th International Conference on Micro Electro Mechanical Systems (MEMS), Paris, France, 29 January-2 February 2012, pp. 281-284.

${ }^{26}$ J. P. Rojas, G. A. Torres Sevilla, M. T. Ghoneim, S. B. Inayat, S. M. Ahmed, A. M. Hussain, and M. M. Hussain, ACS Nano 8, 1468 (2014).

${ }^{27}$ J. P. Rojas, G. A. Torres Sevilla, and M. M. Hussain, Sci. Rep. 3, 2609 (2013).

${ }^{28}$ G. A. Torres Sevilla, S. B. Inayat, J. P. Rojas, A. M. Hussain, and M. M. Hussain, Small 9, 3916 (2013).

${ }^{29}$ G. T. Sevilla, J. P. Rojas, S. Ahmed, A. Hussain, S. B. Inayat, and M. M. Hussain, in 2013 Transducers \& Eurosensors XXVII: The 17th International Conference on Solid-State Sensors, Actuators and Microsystems (TRANSDUCERS \& EUROSENSORS XXVII), Barcelona, Spain, 16 June-20 June 2013 June 2013, pp. 2636-2639. 